\title{
GENERAL INDEX TO VOLUME $67(2010)$
}

Note: Main entries are emboldened; * signifies the page range of keywords; pages with illustrations (Fig.), maps and appendices (App.) are indicated; tables are not annotated separately.

\section{Abbotia Raf., 358 \\ Abutilon}

bidentatum Hochst. ex A.Rich., 206 App. figarianum Webb, 206 App.

fruticosum Guill. \& Perr., 193, 206 App. pannosum (G.Forst.) Schltdl., 194, 206

App.

Acacia Mill., 193-194, 198-199

ehrenbergiana Hayne, 193-194, 196, 198, 205 App.

gerrardii Benth.

var. najdensis Chaudhary, 41, 54 App. tortilis (Forssk.) Hayne, 42, 44 46, 196, 205 App.

subsp. raddiana (Savi) Brenan, 55 App. subsp. tortilis, 193

Acalypha indica L., 205 App.

Acanthaceae, 51 App., 202 App., 231

Acanthonema Hook.f., 177

Achillea L., 247

millefolium L., 241, 246, 253-254

Adelocaryum Brand, 141-154* [145]

anchusoides (Lindl.) Brand, 141-144

capusii (Franch.) Brand, 141, 143

coelestinum (Lindl.) Brand, 141, 143-148

[146], 151-152

coelestinum (Lindl.) R.R.Mill, 141

erythraeum Brand, 141, 144

f. erythraeum, 142

f. subexalata H.Riedl, 142

flexuosum Brand, 141

lambertianum (C.B.Clarke) R.R.Mill, 141, 144-145, 152

malabaricum (C.B.Clarke) Brand, 141, 143-146, 152

nebulicola R.R.Mill, 141, 145-153 [148,

149-150 Figs]

schlagintweitii Brand, 141

Adiantaceae, 51 App.

Adiantum

capillus-veneris L., 45, 51 App.

Aeluropus lagopoides (L.) Trin. ex Thwaites, 194, 207 App.

Aerva

javanica (Burm.f.) Juss. ex Schult., 41, 52

App., 194, 202 App.

Aeschynanthus Jack, 176

bracteatus Wall. ex DC., 163, 175

micranthus C.B.Clarke, 163, 175

Aethionema

carneum (Banks \& Sol.) B.Fedtsch., 53

App.

Africa

Central, 219-233*

South, 353-398*, 425-430*

Agalmyla Blume, 176

biflora (Elmer) Hilliard \& B.L.Burtt, 163, 175

clarkei (Elmer) B.L.Burtt, 163, 175

Agapetes D.Don ex G.Don, 8, 437

Agaricaceae, 405

Agaricus, 405, 409

ephemeroides DC., 406

Agrocybe

fimicola (Speg.) Singer, $\mathbf{4 0 8}$

neocoprophila Singer, 408

Aidia Lour., 265, 283-284

densiflora (Wall.) Masam., 270-271, 273, 284, 341 App., 342 App.

racemosa (Cav.) Tirveng., 271, 273, 284,

341 App., 342 App.

Aizoaceae, 52 App., 202 App.

Aizoon

canariense L., 52 App.

Ajuga

arabica P.H.Davis, 197, 208 App.

Al-AвBASI, T.M., 25-35, 37-56, 189-208

Alcea

striata (DC.) Alef., 42-43, 47, 55 App.

Alectoris

melanocephala Ruppell, 41

Al-Farhan, A., 25-35, 37-56

Al-Harbi, R.J., 37-56, 189-208

Al-Khulaidi, A.W., 25-35

Alliaceae, 52 App. 
Allium

dictyoprasum C.A.Mey. ex Kunth, 52

Aloe App.

vera (L.) Burm.f., 208 App.

Al-Shammari, K.F., 37-56, 189-208

Al-Wetaid, A.H., 37-56, 189-208

Alysicarpus

glumaceus (Vahl) DC., 205 App.

Amaranthaceae, 52 App., 202 App.

Amaranthus

graecizans L., 202 App.

viridis L., 203 App.

Amaryllidaceae, 203 App.

Ammannia

baccifera L., 205 App.

Ammophila, 403

Anacardiaceae, 52 App.

Anamorphic fungi, 420

Anaphalioides (Benth.) Kirp., 236, 248-249

subrigida (Colenso) Anderb., 241, 246, 250

Anaphalis DC., 248-249, 255

javanica Sch.Bip., 242, 246, 250

margaritacea Benth. \& Hook.f., 242, 246, 250

nubigena DC., 242, 246, 250-252

Anarrhinum

forskallii (J.F.Gmel.) Cufod., 56 App.

Ancyclostemon

aureus (Franch.) B.L.Burtt, 163, 175

convexus Craib, 163, 175

Andrachne

aspera Spreng.

var. glandulosa Hochst. ex A.Rich., 206 App.

Anellaria

semiovata (Sowerby) A.Pearson \&

Dennis, 409

Anisosciadium

lanatum Boiss., 42, 56 App.

Anna

mollifolia (W.T.Wang) W.T.Wang \&

K.Y.Pan, 163, 175

submontana Pellegr., 164, 175

Anogeissus

dhofarica A.J.Scott, 151-152

Antennaria Gaertn., 236, 248-249, 252-255

anaphaloides Rydb., 242, 246, 250 carpatica (Wahlenb.) Bluff \& Fingerh., 242, 246, 250, 252

dioica (L.) Gaertn., 242, 246, 249-252, 254

luzuloides Torr. \& Gray, 242, 246, 252 253

Anticharis

glandulosa Asch., 208 App.

Anvillea

garcinii (Burm.f.) DC., 44, 52 App.

Aoranthe Somers, 267, 285

Apiaceae see Umbelliferae

Apocynaceae, 1-5, 52 App., 203 App.

subfam. Apocynoideae, 1-5*

tribe Mesechiteae, 1

Arabian Peninsula, Important Plant Areas (IPAs) in, 25-35*, 37-56*, 189-208

Arctic, 7-24*

Arecaceae see Palmae

Argyrolobium

arabicum (Decne.) Jaub. \& Spach, 205 App.

Aristida adscensionis L., 207 App.

funiculata Trin. \& Rupr., 207 App.

Aristolochia

bracteolata Lam., 203 App.

Aristolochiaceae, 203 App.

Armeria macloviana Cham., 403

Artemisia, 258 herba-alba Asso, 52 App.

sieberi Besser, 41, 52 App.

Arthraxon inermis Hook.f., 147

purandharensis Bharucha, 147

Arthrocnemum macrostachyum (Moric.) K.Koch, 194, 196 Fig., 203 App.

Arundinella, 448

Asclepiadaceae, 52 App.

Ascobolaceae, 412

Ascobolus, 412-413

albidus P.Crouan \& H.Crouan, 412

brantophilus Dissing, 412

degluptus Brumm., 412-413

furfuraceus Pers., 412

hawaiiensis Brumm., 412

immersus Pers., 412

michaudii Boud., 413 
stictoideus Speg., 412-413

Ascomycota, 399-423* [411]

Asellia

patrizii DeBeaux, 190

Asia, Southeast, 75-121*

Asparagaceae, 52 App., 203 App.

Asparagus

aphyllus L., 52 App.

flagellaris (Kunth) Baker, 193, 203 App.

Asphodelaceae, 52 App.

Asphodelus

tenuifolius Cav., 42, 46, 52 App.

Asteraceae, 203 App., 235-264*, 400-401

tribe Anthemideae, 246, 248-249, 252 254

tribe Astereae, 246, 248-249, 252-254

tribe Gnaphalieae, 235-264*

see also Compositae

Asteriscus

pygmaeus (DC.) Coss. \& Durieu, 44, 52 App.

Astracantha

echinus (DC.) Podlech

subsp. arabica Hedge \& Podlech, 43, 47, 55 App.

Astragalus L., 208 App.

spinosus (Forssk.) Muschl., 44, 55 App.

Atractocarpus Schltr. \& K.Krause, 265342* [272 Fig.]

crosbyi (Burkill) Puttock, 267

heterophyllus (Montrouz.) Guillaumin \& Beauvis., 265, 270-272 \& Fig., 274, 276, 278, 284, 341 App., 342 App.

tenuiflorus (A.C.Sm.) Puttock, 267

Atractocarpus sensu Puttock, 285

Atractylis carduus (Forssk.) C.Chr., 52 App.

Atriplex

farinosa Forssk., 203 App.

halimus L., 203 App.

Austin, D.F., 467-468

Auvray, G., 451-465

Avery, T.S., 7-24

Avicennia, 197 marina (Forssk.) Vierh., 192, 197, 202 App.

Baccharis, 401

Balaenoptera acutorostrata Lacépède, 190

Ballota undulata (Sieber ex Fresen.) Benth., 42, 54 App.

Barbeya

oleoides, 31

Barbeyaceae, 31

Barleria hochstetteri Nees ex DC., 202 App.

Basilicum polystachyon (L.) Moench, 194, 197, 205 App.

Basidiomycota, 399-423* [404]

Begonia, 123-140* sect. Diploclinium, 123-140 [126 Maps] sect. Platycentrum, 124 sect. Reichenheimia, 131 acclivis C.Coyle, 123-128 [125, 126 Map,

127 Fig.], 136

acuminatissima Merr., 134

anisoptera Merr., 124, 136

cleopatrae C.Coyle, 123-124, 126 Map,

127 Fig., 128-129, 136

concanensis A.DC., 147

coronensis, 124, 126 Map, 127 Fig., 129-

131,140

gueritziana Gibbs, 124, 138-139

gutierrezii C.Coyle, 123-124, 126 Map,

127 Fig., 131-132

hernandioides Merr., 132

longiscapa Warb., 134

mindorensis Merr., 123, 125-126 Map,

132-134 [133 Fig.]

nigritarum (Kamel) Steud., 123, 135

palawanensis, 131

pinamalayensis Merr., 132, 134

rhombicarpa A.DC., 134-135

rubiteae M.Hughes, 123, 125-126 Map,

133 Fig., 134-135

sordidissima Elmer, 134

suborbiculata Merr., 124-126 Map, 128,

131, 133 Fig., 135

tayabensis Merr., 132

wadei Merr., 124, 126 Map, 131, 133 Fig.,

136-137

wilkiei C.Coyle, 123-126 Map, 138-139

Fig.

woodii Merr., 124, 126 Map, 130, 139 \&

Fig.

Begoniaceae, 123-140

Bhutan, 451-465

Biodiversity, 399-423* 
Biogeography

of Porterandia, 265-342*

Biscutella

didyma L., 53 App.

Bistella

digyna (Retz.) Bullock, 208 App.

Blechnum

magellanicum Desv., 403

Blepharis

ciliaris (L.) B.L.Burtt, 51 App., 193, 202

App.

BцÖCH, C., 235-264

Boea

hygrometrica (Bunge) R.Br., 164, 175

magellanica Lam., 164, 175

Boehmeria Jacq., 431-450*

densiflora Hook. \& Arn., 449

erythropoda auct. non Miq., 441

erythropoda Miq., 444

hamiltoniana Wedd., 443, 445

heterophylla Wedd., 440

leptostachya Friis \& Wilmot-Dear, 431450* [441, 442-443 Fig., 444 Map]

listeri Friis \& Wilmot-Dear, 431-450*

[432, 433 Fig., 434 Map]

macrophylla D.Don

var. listeri sensu King, 437

manipurensis Friis \& Wilmot-Dear, 431450* [434 Map, 438, 439 Fig.]

multiflora C.B.Rob., 449

penduliflora Wedd. ex D.G.Long, 436437, 449

pilosiuscula (Blume) Hassk., 436

subintegra Friis \& Wilmot-Dear, 431450* [444, 446 Fig., 447 Map]

zollingeriana Wedd., 443, 445

Boeica C.B.Clarke, 177

ferruginea Drake, 164, 175

porosa C.B.Clarke, 164, 175

Boerhavia

diffusa L., 206 App.

Boesenbergia Kuntze, 75, 77, 97

anomala (Hallier f.) Schltr., 85

biloba (Ridl.) Schltr., 89

bractescens (Ridl.) Merr., 112

calophylla (Ridl.) Schltr., 89

concinna (Ridl.) Schltr., 95

gracilipes (K.Schum.) R.M.Sm., 97, 118

klossii (Ridl.) Loesen., 98

lancifolia (Ridl.) Schltr., 105 laxiflora (Valeton) Loesen., 112

malaccana (Baker) Govaerts, 105

minor (Baker) Kuntze, 90

oculata (Ridl.) Schltr., 108

reticosa (Ridl.) Merr., 113

scaphochlamys (Ridl.) Schltr., 105

Bolbitiaceae, 408

\section{Bolbitius}

boltonii (Pers.) Fr., 408

flavidus (Bolton) Massee, 408

fragilis (L.) Fr., 408

vitellinus Pers., 408

Bolivia, 69-73*

Bombardioidea

stercoris (DC.) N.Lundq., 416

Boraginaceae, 52 App., 141-154*, 203 App. tribe Cynoglosseae, 141-154

Boreal zone, 7-24*

Borneo, 75-121, 265-342* [330 Map]

Brachiaria ramosa (L.) Stapf, 207 App.

Brandella R.R.Mill, 141-154* erythraea (Brand) R.R.Mill, 141-142, 144

f. subexaltata (H.Riedl) R.R.Mill, 141142

Brassicaceae see Cruciferae

Brazil, Northeast, 1-5*

BReteler, F.J., 343-346

Briggsia longipes (Hemsl. ex Oliv.) Craib, 164, 175 mihieri Craib, 164, 175 muscicola (Diels) Craib, 164, 175 rosthornii (Diels) B.L.Burtt, 164, 175

Bromus fasciculatus C.Presl, 42, 54 App.

Bungarimba K.M.Wong, 265-342* [272 Fig.] kahayanensis K.M.Wong, 271, 284, 341 App., 342 App.

ridsdalei K.M.Wong, 270-272 \& Fig., 276

Fig., 281, 284, 341 App., 342 App.

sessiliflora (Ridl.) K.M.Wong, 271, 284, 327, 341 App., 342 App.

Burbidgea Hook.f., 65

Burma, 451-465

Burseraceae, 203 App.

Caatinga, 1-5*

Cadaba

farinosa Forssk., 203 App. glandulosa Forssk., 203 App. 
longifolia DC., 203 App.

rotundifolia Forssk., 203 App.

Calcareoboea

coccinea C.Y.Wu ex H.W.Li, 164, 175

Calotropis

procera (Aiton) Aiton f., 203 App.

Cambodia, 155-160*

Campanulaceae, 425-430*

Camptandra Ridl., 75

Canis

lupus arabs Pocock, 40

Cannabis, 281

Canscora, 155-160*

alata (Roth) Wall., 160

andrographioides auct., 158

andrographioides Griff. ex C.B.Clarke, 158,160

bidoupensis Hul, 155, 158-160 [159 Fig.] diffusa (Vahl) R.Br. ex Roem. \& Schult., 160

Cape Floristic Region, 353-398*

Cape Peninsula, 353-398*

Capparaceae, 52 App., 203 App.

Capparis

aphylla Hayne ex Roth, 203 App.

cartilaginea Decne., 203 App.

decidua (Forssk.) Edgew., 203 App.

spinosa L., 44, 52 App., 194, 204 App.

Capra

ibex nubiana F.Cuvier, 38

Caracal

caracal schmitzi Matschie, 40

Caralluma

acutangula (Decne.) N.E.Br., 203 App. adscendens (Roxb.) Haw., 203 App.

edulis (Edgew.) Benth. ex Hook.f., 203

App.

Carpodacus

synoicus Temminck, 41

Carpolobia G.Don, 343-346*

alba G.Don, 343, 346

gabonica Breteler, 343-346 [344 Fig., 345

Map]

goetzei Gürke, 343

gossweileri (Exell) E.M.A.Petit, 343, 346

lutea G.Don, 343

Caryophyllaceae, 52 App., 204 App.

Castroviejoa Galbany, L.Sáez \& Benedi, 248-249, 255 frigida (Labill.) Galbany, L.Sáez \&

Benedi, 242, 246, 250

montelinasana (Em.Schmid) Galbany,

L.Sáez \& Benedi, 243, 246, 250

Cautleya (Royle ex Benth.) Hook.f., 451$465 *[454]$

cathcartii Baker, 452, 454, 458, 460

gracilis (Sm.) Dandy, 452-455, 460, 464 var. gracilior (K.Schum.) Sanjappa, 455

var. gracilis, 455-456 Map, 464

var. robusta (K.Schum.) Sanjappa, 454, 458-460 [459 Fig., 460 Map]

lutea (Royle) Hook.f., 451, 453, 455

var. gracilior K.Schum., 452, 455

var. robusta K.Schum., 452, 454, 458460

petiolata Baker, 452-453, 462-464 [463

Fig.]

robusta Baker, 452, 454, 462

spicata (Sm.) Baker, 452-455, 460-462

[461 Fig., 462 Map], 464

Cavendishia Lindl., 8

Caylusea

hexagyna (Forssk.) M.L.Green, 41, 55

App.

Celastraceae, 204 App.

Cenchrus

ciliaris L., 207 App.

pennisetiformis Hochst. \& Steud., 54

App.

setigerus Vahl, 207 App.

Centaurea

eryngioides Lam., 44, 52 App.

pseudosinaica Czerep., 41, 52 App.

sinaica DC., 44, 52 App.

Central Africa, 219-233*

Ceratostomataceae, 417

Cerrado, 69-73*

Chaetomiaceae, 415

Chaetomium, 415

crispatum (Fuckel) Fuckel, 415

Chaetosciadium

trichospermum (L.) Boiss., 46-47, 56 App.

Chascanum

marrubiifolium Fenzl ex Walp., 208 App.

Chassalia Comm. ex Poir., 219-233*

corallifera (A.Chev. ex De Wild.) Hepper, 220, 224

ischnophylla (K.Schum.) Hepper, 224 
kolly (Schumach.) Hepper, 220, 224

lutescens O.Lachenaud \& D.J.Harris,

219-224 [220, 221 Fig., 222 Map]

subherbacea (Hiern) Hepper, 224

subspicata K.Schum., 224

Chazaliella E.M.A.Petit \& Verdc., 219, 228

letouzeyi Robbr., 228

oddonii (De Wild.) E.M.A.Petit \& Verdc. var. cameroonensis Verdc., 220

Cheilymenia, 412

coprinaria (Cooke) Boud., 411

var. megaspora Gamundi, 411

fimicola (de Not. \& Bagl.) Dennis, 411

humarioides Gamundi, 412

raripila (Phill.) Dennis, 411

stercorea (Pers.) Boud., 411

Chelonia

mydas L., 191

Chen, W.-H., 161-184

Chenolea

diffusa Thunb., 393

Chenopodiaceae, 52 App.

Chiliadenus

montanus (Vahl) Brullo, 43, 52 App.

Chiliotrichum

diffusum (G.Forst.) Kuntze, 400

China, 161-184, 431-450*, 451-465

Chirita Buch.-Ham. ex D.Don, 177

asperifolia (Blume) B.L.Burtt, 164, 175176

caliginosa C.B.Clarke, 164, 175

lavandulacea Stapf, 164, 175

pinnata W.T.Wang, 164, 175

pinnatifida (Hand.-Mazz.) B.L.Burtt, 164, 175

pumila D.Don, 165, 175

sinensis Lindl., 165, 175

urticifolia Buch.-Ham. ex D.Don, 165, 175

walkeri Gardner, 165, 175

Chiritopsis

repanda W.T.Wang

var. guilinensis W.T.Wang, 165, 175

Chloris

barbata (L.) Sw., 207 App.

Chrozophora

oblongifolia (Delile) A.Juss. ex Spreng., 53 App., 205 App.

Chrysopogon

plumulosus Hochst., 207 App.
Cibirhiza P.Bruyns, 152

Cinchona, 437

Circumscription

of Porterandia, 265-342

Cissus

quadrangularis L., 193, 208 App.

rotundifolia (Forssk.) Vahl, 208 App.

Cistaceae, 52 App.

Cistanche

phelypaea (L.) Cout., 46, 55 App. tubulosa (Schenk) Hook.f., 206 App.

Citrullus colocynthis (L.) Schrad., 53 App., 204 App.

Cleistanthus, 231

Cleome brachycarpa Vahl ex DC., 204 App.

gynandra L., 204 App.

noeana Boiss. \& Popov subsp. brachystyla (Deflers)

D.F.Chamb. \& Lamond, 194, 197, 204 App.

tenella L.f., 204 App.

Clitocybe

amarescens Harmaja, 404

harmajae Lamoure, 405

metachroa (Fr.) P.Kumm., 404

nitrophila Bon, 405

Clitopilus

hobsonii (Berk. \& Broome) P.D.Orton

var. chilensis Singer, 410

passeckerianus (Pilát) Singer, 410

Clustering strategies, 7, 13-14, 16-17

Cocculus

pendulus (J.R.Forst. \& G.Forst.) Diels, 206 App.

Coffea L., 273

subgen. Baracoffea (J.-F.Leroy)

J.-F.Leroy, 273

subgen. Coffea, 273

Commelina

benghalensis L., 204 App.

forsskalii Vahl, 204 App.

Commelinaceae, 204 App.

Commicarpus

helenae (Roem. \& Schult.) Meikle, 206 App.

Commiphora

erythraea (Ehrenb.) Engl., 194, 197, 200, 203 App. 
gileadensis (L.) C.Chr., 193, 203 App.

Compositae, 52 App.

see also Asteraceae

Conandron

ramondioides Siebold \& Zucc., 165, 175

Congo Brazzaville, 343-346* [345 Map]

Coniochaeta

discospora (Auersw.) Cain, 415

hansenii (Oudem.) Cain, 415

leucoplaca (Berk. \& Ravenel) Cain, 415

ligniaria (Grev.) Massee, 415

saccardoi (Marchal) Cain, 415

scatigena (Berk. \& Broome) Cain, 415

Coniochaetaceae, 415

Conocybe

cryptocystis (Atk.) Singer, 408

cryptocystis auct., 408

digitalina (Velen.) Singer, 408

magnispora (Murrill) Singer, 408

pubescens (Gillet) Kühner, 408

subpubescens P.D.Orton, 408

Conservation

in Arabian Peninsula, 25-35*, 37-56*

of Paraboea, 209-217*

in Saudi Arabia, 189-208*

Convolvulaceae, 53 App., 204 App., 467468

Convolvulus

arvensis L., 204 App.

glomeratus Choisy, 204 App.

hystrix Vahl, 53 App.

pilosellifolius Desr., 204 App.

rhyniospermus Hochst. ex Choisy, 204

App.

Coprinaceae, 405, 407

Coprinellus

brevisetulosus (Arnolds) Redhead, Vilgalys \& Moncalvo, 405

curtus (Kalchbr.) Vigalys, Hopple \& Jacq.Johnson, 405-406

heptemerus (M.Lange \& A.H.Sm.)

Vigalys, Hopple \& Jacq.Johnson, 405

pellucidus (P.Karst.) Redhead, Vigalys \& Moncalvo, 405-406

Coprinopsis, 406 cordispora (T.Gibbs) Watling \& M.J.Richardson, 399, 406 ephemeroides (DC.) Watling \& M.J.Richardson, 399, 406 nivea (Pers.) Redhead, Vigalys \&

Moncalvo, 406-407

pachysperma (P.D.Orton) Redhead,

Vigalys \& Moncalvo, 407

radiata (Bolton: Fr.) Redhead, Vigalys \&

Moncalvo, 407

stercorea (Fr.) Redhead, Vigalys \&

Moncalvo, 407

vermiculifera (Joss. ex Dennis) Redhead,

Vigalys \& Moncalvo, 407

Coprinus, 405, 407

brevisetulosus Arnolds, 405

bulbillosus Pat., 406

comatus (O.F.Müll.) Pers., 405

cordisporus T.Gibbs, 406

curtus Kalchbr., 405

ephemeroides (DC.) Fr., 406

heptemerus M.Lange \& A.H.Sm., 405

latisporus P.D.Orton, 406

miser P.Karst., 407

niveus (Pers.) Fr., 406

pachyspermus P.D.Orton, 407

patouillardii Quél., 406

pellucidus P.Karst., 406

plicatiloides Buller, 405

radiatus (Bolton) Gray, 407

stellatus Sacc., 405

stercorarius (Bull.) Fr., 407

stercoreus Fr., 407

vermiculifer Joss. ex Dennis, 407

\section{Coprobia}

granulata (Bull.) Boud., 412

Coprophilous fungi, of the Falkland Islands, 399-423 [401 Map]

Corallodiscus Batalin, 171, 174, 177

lanuginosus (Wall. ex R.Br.) B.L.Burtt, 165,175

Corchorus

depressus (L.) Stocks, 206 App.

olitorius L., 206 App.

trilocularis L., 206 App.

Cornulaca ehrenbergii Asch., 203 App.

Cortaderia pilosa (d'Urv.) Hack., 403

Cotula

coronopifolia L., 391

scariosa (Cass.) Franch., 403

Coyle, C., 123-140

Crassulaceae, 53 App. 
Craterocapsa, 425

Cressa cretica L., 204 App.

Crotalaria microphylla Vahl, 205 App. persica (Burm.f.) Merr., 55 App.

Crucianella ciliata Lam., 55 App. membranacea Boiss., 55 App.

Cruciferae, 47, 53 App.

Cucumis melo L., 204 App. subsp. agrestis (Naudin) Pangalo, 204 App.

prophetarum L., 193, 204 App. var. dissectus (Naudin) C.Jeffrey, 53 App.

Cucurbitaceae, 53 App., 204 App.

Cupido, C.N., 425-430

Cupressaceae, 53 App.

Curcuma, 79 sect. Hitcheniopsis Baker, 79 sect. Mesantha Horan., 79

kunstleri Baker, 79, 101 var. rubra Ridl., 102 lanceolata Ridl., 109 sylvestris Ridl., 79, 116

Cuscuta planiflora Ten., 204 App.

Cycnogeton Endl., 358

Cymbopogon schoenanthus (L.) Spreng., 42-43, 54 App. Cymodocea rotundata Asch. \& Schweinf., 195, 204 App. serrulata (R.Br.) Asch. \& Magnus, 195, 204 App.

Cymodoceaceae, 204 App.

Cynodon dactylon (L.) Pers., 207 App.

Cynoglossum L., 141-143 subgen. Eleutherostylum (Brand) H.Riedl, 145 sect. Paracaryopsis H.Riedl, 144-145 anchusoides Lindl., 142 coelestinum I.M.Johnston, 152 coelestinum Lindl., 142, 144-147 longiflorum Benth., 143 malabaricum (C.B.Clarke) H.Riedl, 142, 152

Cyperaceae, 204 App.
Cyperus

bulbosus Vahl, 204 App.

conglomeratus Rottb., 194-195, 204 App.

jeminicus Rottb., 208 App.

rubicundus Vahl, 204 App.

Cypholophus Wedd., 432, 449

Cyrtandra

cupulata Ridl., 165, 175

glabra Banks ex Gaertn., 165, 175

longifolia (Wawra) Hillebr. ex

C.B.Clarke, 165, 175

pendula Blume, 165, 175

\section{Dactyloctenium}

aegyptium (L.) Willd., 207 App.

aristatum Link, 207 App.

scindicum Boiss., 207 App.

Dalechampia

scandens $\mathrm{L}$.

var. cordofana (Hochst. ex Webb)

Müll.Arg., 205 App.

Deconica subcoprophila (Britzelm.) E.Horak, 411

Delitschia niesslii Oudem., 419

Delphinium sheilae Kit Tan, 42-43, 47, 55 App.

Description of Boehmeria, 431-450*

Desmostachya bipinnata (L.) Stapf, 193, 207 App.

Dhofaria A.G.Mill., 152

Dianthus strictus Banks \& Sol., 52 App.

Dichanthium foveolatum (Delile) Roberty, 207 App.

Dickoré, W.B., 235-264

Didymocarpoid Gesneriaceae, 161-184

Didymocarpus Wall., 171, 173, 176 antirrhinoides A.Weber, 165, 175 citrinus Ridl., 165, 175 cordatus Wall. ex DC., 165, 175 podocarpus C.B.Clarke, 166, 175 purpureobracteatus W.W.Sm., 166, 175 stenanthos C.B.Clarke, 166, 175

Didymostigma W.T.Wang, 178

Digera muricata (L.) Mart., 203 App.

Digitaria ciliaris (Retz.) Koeler, 207 App. 


\section{Dinebra}

retroflexa (Vahl) Panz, 207 App.

Dipcadi Medik., 205 App.

Diplotaxis harra (Forssk.) Boiss., 53 App.

Dipsacaceae, 53 App.

Dipterygium glaucum Decne., 204 App.

Disjunction, 141-154*

Distichochlamys M.F.Newman, 75, 77, 347

DNA markers, 235-264

Dolicholoma D.Fang \& W.T.Wang, 171172, 176, 178

jasminiflorum D.Fang \& W.T.Wang, 166, 175

Dolichorhynchus

arabicus, 46

Dothideales, 419

Douepea arabica (Hedge \& Kit Tan) O.Appel \& Al-Shehbaz, 46-47, 48, 53 App.

Drake-Brockmania somalensis (Hack.) Stapf, 197, 207 App.

Dugong dugon Müller, 190

\section{Ecbolium} viride (Forssk.) Alston, 202 App.

Echinops glaberrimus DC., 46-47, 53 App. hystrichoides Kit Tan, 41, 53 App.

Echium rauwolfii Delile, 44, 52 App.

Ecology of Triglochin, 353-398*

Edelweiss, molecular phylogeny of, 235-264

Elionurus royleanus Nees ex A.Rich., 207 App.

Elymus, 403

Empetrum rubrum Vahl, 401

Endemism in Paraboea, 209

Enhalus acoroides (L.f.) Royle, 205 App.

Entolomataceae, 410

Ephedra aphylla Forssk., 53 App. pachycalda Boiss. subsp. sinaica (H.Riedl) Freitag \&

Majer-Stolte, 43, 47, 53 App.

Ephedraceae, 53 App.

Epipactis veratrifolia Boiss. \& Hohen., 45-47, 55

App.

Epitype, 185-187*

Eragrostis ciliaris (L.) R.Br., 207 App.

lepida (A.Rich.) Hochst. ex Steud., 207 App.

minor Host, 207 App.

Eremobium aegyptiacum (Spreng.) Asch. \& Schweinf. ex Boiss., 53 App.

Eretmochelys imbricata L., 191

Erica arborea L., 190, 208 App.

Ericaceae, 7-24*, 401 tribe Vaccinieae, 8

Eriochloa fatmensis (Hochst. ex Steud.) Clayton, 207 App.

Erodium, 44 hirtum (L.) Willd., 44, 53 App. malacoides (L.) L'Hér., 44, 53 App. neuradifolium Delile ex Godr., 44, 53 App. oxyrhynchum M.Bieb., 44, 53 App.

Euphorbia balsamifera Aiton, 151 collenetteae Al-Zahrani \& El-Karemy, 194-195 \& Fig., 197, 205 App. fractiflexa S.Carter \& J.R.I.Wood, 205 App. granulata Forssk., 205 App.

Euphorbiaceae, 53 App., 205 App.

Evolvulus alsinoides (L.) L., 204 App.

Ewartia Beauverd, 248-249, 252-253, 255 argentifolia N.A.Wakef., 243, 246, 250 catipes (Hook.f.) Beauverd, 243, 246, 250-254 meredithiae (F.Muell.) Beauverd, 243, 246, 250 planchonii (Hook.f.) Beauverd, 243, 246, 250

Ewartiothamnus Anderb., 248-249 sinclairii (Hook.f.) Anderb., 243, 246, 250 Exacum, 155-160* 
darae Hul, 155-157 \& Fig. pteranthum Wall. ex G.Don, 157-158 sutaepense auct., 156 sutaepense Hosseus ex Craib, 155-157

var. gracile Toyokuni, 155

f. gracile (Toyokuni) Toyokuni, 155 tetragonum Roxb., 158

External transcribed spacer (ETS), 235-264*

Fabaceae see Leguminosae

Fagonia

indica Burm.f., 56 App.

mollis Delile, 44, 56 App.

ovalifolia Hadidi, 46, 56 App.

tristis Sickenb., 42, 56 App.

Falkland Islands, coprophilous fungi of, 399-423 [401 Map]

Farasan Archipelago, Saudi Arabia, 189-

Farsetia 208* [190 Fig.]

aegyptia Turra, 44, 53 App.

Felix sylvestris tristrami Pocock, 40

Ferula

ovina Boiss., 41, 56 App.

rutbaensis C.C.Towns., 41, 56 App.

sinaica Boiss., 56 App.

Ficus

cordata Thunb.

subsp. salicifolia (Vahl) C.C.Berg, 43, 45-46, 55 App., 206 App.

glumosa Delile, 206 App.

palmata Forssk.

subsp. virgata (Roxb.) Browicz, 45, 55 App.

populifolia Vahl, 196-197, 200, 206 App. vasta Forssk., 151

Filago L., 248-249, 255

pyramidata L., 243, 246, 250

Fioria

dictyocarpa (Webb) Mattei, 206 App.

Flora

of China, 161-184*

of Pakistan, 185-187

Flueggea

leucopyrus Willd., 197, 206 App.

virosa (Roxb. ex Willd.) Royle, 206 App.

Forsskaolea

tenacissima L., 56 App.

viridis Ehrenb. ex Webb, 208 App.
FRIIS, I., 431-450

Frost, 7-24*

Fungi, coprophilous, 399-423

Gabon, 343-346* [345 Map]

Gamochaeta Wedd., 248-249, 252-253

pensylvanica (Willd.) Cabrera, 243, 246, 254

spicata (Lam.) Cabrera, 244, 246, 250

Gardenia auct. non Ellis, 285

Gardenia Ellis, 267, 269

affinis Valeton ex Winkl., 313

anisophylla Jack ex Roxb., 268, 285, 290, 293

var. macroptera (Miq.) Valeton ex

Winkl., 268, 312

var. polyneura Vahl ex Winkl., 268, 305

var. subsessilis auct. non Valeton ex

Winkl., 307

var. subsessilis Valeton ex Winkl., 268, 325

macroptera Miq., 312

Gastrochilus D.Don, 79

Gastrochilus Wall., 79

anomalum (Hallier f.) K.Schum., 85

biloba Ridl., 79, 88

var. lanceolata Ridl., 89-90

bractescens Ridl., 112

calophylla Ridl., 89

concinna (Baker) Ridl., 79, 95

hallieri (Hallier f.) Ridl., 85, 87

klossii Ridl., 98

kunstleri (Baker) Valeton, 101

lanceolata Ridl., 103

lancifolius Ridl., 79, 105

laxiflorum Valeton, 112

longifolia Ridl., 90

minor Baker, 90

minor sensu Ridl., 89

oculata Ridl., 79, 108

reticosa Ridl., 112

scaphochlamys Ridl., 79, 105

subbilobus Ridl., 115

Gaultheria, 403

pumila (L.f.) D.J.Middleton, 401

Gazella

gazella Pallas, 40, 193

Gentiana

khammouanensis Hul, 155

Gentianaceae, 155-160*

Geraniaceae, 53 App. 
Gesneriaceae, 161-184*, 209-217* subfam. Didymocarpoideae Endl., 178 tribe Didymocarpeae Endl., 178

Gilbertiodendron dewevrei (De Wild.) J.Léonard, 227, 231

Ginger, 75-121*

Globularia arabica Jaub. \& Spach, 43, 47, 53 App.

Globulariaceae, 53 App.

Glossonema boveanum (Decne.) Decne., 203 App.

Gnaphalium L., 248-249, 252, 254-255 hoppeanum (L.) Hilliard \& B.L.Burtt, 244, 246, 250

norvegicum Gunnerus, 244, 246, 250

supinum L., 244, 246, 250

sylvaticum L., 244, 246, 249-251

Gomphocarpus sinaicus Boiss., 41, 46, 52 App.

Gossypium hirsutum L., 206 App.

Gramineae, 54 App. see also Poaceae

Grewia erythraea Schweinf., 206 App. tenax (Forssk.) Fiori, 193, 197, 206 App.

Gunnera, 403

Gymnocarpos decandrus Forssk., 43-44, 52 App.

Gynandriris sisyrinchium (L.) Parl., 54 App.

Gynandropsis gynandra (L.) Briq., 204 App.

Gyps fulvus Hablizl, 41

Haberlea Friv., 174, 177 rhodopensis Friv., 162, 166, 175

Hall, M., 25-35, 37-56, 189-208

Halodule uninervis (Forssk.) Boiss., 195, 204 App.

Halopeplis perfoliata (Forssk.) Bunge ex Asch. \& Schweinf., 194, 196 Fig., 203 App.

Halophila ovalis (R.Br.) Hook.f., 195, 205 App. stipulacea (Forssk.) Asch., 195, 205 App.

Halopyrum mucronatum (L.) Stapf, 207 App.

Haloragaceae, 54 App.
Haloxylon, 46 salicornicum (Moq.) Boiss., 42, 44, 46, 52 App.

Hamiyah in Arabian Peninsula, 29

Haplochorema K.Schum., 75, 77

gracilipes K.Schum., 97

petiolatum K.Schum., 110

polyphyllum K.Schum., 112

Haplophyllum tuberculatum (Forssk.) A.Juss., 56 App.

HARRIS, D.J., 219-233

Hebe, 403

Helianthemum lippii (L.) Dum.Cours., 42-43, 52 App.

Helichrysum Mill., 236, 248-249 leucopsideum DC., 244, 246, 251-252

Heliotropium longiflorum (A.DC.) Jaub. \& Spach, 203 App. ramosissimum (Lehm.) Sieber ex A.DC., 52 App., 203 App. strigosum Willd., 203 App. zeylanicum (Burm.f.) Lam., 203 App.

Hemiboea bicornuta (Hayata) Ohwi, 166, 175 cavaleriei H.Lév., 166, 175 gracilis Franch., 166, 175 subcapitata C.B.Clarke, 166, 175

Hexaglochin Nieuwl., 358 maritima (L.) Nieuwl., 358

Hibiscus micranthus L.f., 206 App.

Himalaya, 57-64*, 235-264, 431-450*

Himas in Arabian Peninsula, 29-30, 48 on Farasan Islands, 198

Hitcheniopsis (Baker) Ridl., 79 kunstleri (Baker) Ridl., 79, 101 var. rubra (Ridl.) Ridl., 102 lanceolata (Ridl.) Ridl., 79, 109 sylvestris (Ridl.) Ridl., 79, 116

Holcus lanatus L., 403

Huaylla, H., 69-73

Hughes, M., 123-140

HuL, S., 155-160

Hyacinthaceae, 205 App.

Hyaena

hyaena sultana Pocock, 40

Hydrocharitaceae, 205 App. 
Hymenocoleus Robbr., 228

axillaris Robbr., 228

Hyoscyamus

aureus L., 56 App.

pusillus L., 56 App.

Hyparrhenia

hirta (L.) Stapf, 44, 54 App., 207 App.

Hypericaceae, 54 App.

Hypericum sinaicum Hochst. ex Boiss., 43, 47, 54 App.

Hyphaene

thebaica (L.) Mart., 45 \& Fig., 55 App., 193, 196, 206 App.

Hypocopra, 418

brefeldii (Zopf) Zopf, 418

merdaria (Fr.) J.Kickx f., 418

planispora J.C.Krug \& Cain, 418

stephanophora J.C.Krug \& Cain, 418

Hypocreales, 417-418

Hystrix

indica Kerr, 41

Imperata

cylindrica (L.) Raeusch., 54 App.

Important Plant Areas (IPAs), 25-35*, 37$56^{*}, 189-208^{*}$

India, 57-64*, 141-154*, 451-465

Indigofera

caerulea Roxb., 205 App.

hochstetteri Baker, 205 App.

linifolia (L.f.) Retz., 205 App.

oblongifolia Forssk., 193-194, 205 App.

semitrijuga Forssk., 205 App.

spinosa Forssk., 205 App.

volkensii Taub., 208 App.

Indo-China, 155-160

Indonesia, 65-68*, 347-352 [349 Map]

Internal transcribed spacer (ITS), 235-264*

Iodophanus

carneus (Pers.) Korf, 414

testaceus (Moug. ex Fr.) Phill., 414

Iphiona

mucronata (Forssk.) Asch. \& Schweinf., 43, 53 App.

Ipomoea

bernoulliana Peter, 467-468

eriocarpa R.Br., 204 App.

hochstetteri House, 193-194, 197, 204 App.

obscura (L.) Ker-Gawl., 193, 204 App.

santae-rosae Standl. \& Steyerm., 467 sinensis (Desr.) Choisy

subsp. blepharosepala (A.Rich.)

Meeuse, 208 App.

Iridaceae, 54 App.

Isatis

lusitanica L., 53 App.

IUCN conservation assessment, 353-398*

IUCN Red List, 123-140*

Jabal Qaraqir, Saudi Arabia, 37-56 [39 Map]

Jancaea Boiss., 176-177

heldreichii Boiss., 162, 166, 175

Jatropha

glauca Vahl, 205 App.

Julbernardia, 231

Juncaceae, 205 App.

Juncaginaceae, 353-398*

Juncago Ség., 358

chabraei Bubani, 363

Juncago Tourn. ex Moench, 358

Juncus, 389

acutus L., 363

rigidus Desf., 205 App.

Juniperus

phoenicea L., 43, 53 App.

Justicia

flava (Vahl) Vahl, 194, 202 App.

KAdereit, J.W., 353-398

Kaempferia L., 75, 77, 79

anomala Hallier f., 79, 85

biloba (Ridl.) K.Schum., 89

calophylla (Ridl.) K.Schum., 89

concinna Baker, 79, 94

cumingii K.Schum., 105-106

lancifolia (Ridl.) K.Schum., 105

lutea C.H.Wright, 101

malaccana (Baker) K.Schum., 105

Kailarsenia Tirveng., 269

Kaisupeea

herbacea (C.B.Clarke) B.L.Burtt, 166, 175

Kalimantan, Indonesia, 347-352* [349 Map]

Kedrostris

gijef (Forssk. ex J.F.Gmel.) C.Jeffrey, 204 App.

Key Biodiversity Area (KBA), 25-35*, 37

Kickxia

acerbiana (Boiss.) Täckh. \& Boulos, 56 App. 
corallicola D.A.Sutton, 193-194, 197, 206 App.

hastata (R.Br. ex Benth.) Dandy, 206 App.

Kiew, R., 209-217

KöcKe, A.V., 353-398

Kohautia caespitosa Schnizl., 208 App.

Labiatae, 54 App., 452 see also Lamiaceae

Lachenaud, O., 219-233

Lamiaceae, 205 App. see also Labiatae

Lappula spinocarpa (Forssk.) Asch. ex Kuntze, 52 App.

Lasiobolus ciliatus (J.C.Schmidt) Boud., 414 cuniculi Velen., 414 equinus (O.F.Müll.) P.Karst., 414 var. pilosus (Fr.) Sacc., 414 papillatus (Pers.) Sacc., 414 pilosus (Fr.) Sacc., 414 ruber (Quél.) Sacc., 414

Lasiosphaeriaceae, 416

Laudakia stellio brachydactyla Haas, 41

Launaea intybacea (Jacq.) Beauverd, 203 App. procumbens (Roxb.) Ramayya \& Rajagopal, 203 App. spinosa (Forssk.) Sch.Bip., 42, 53 App.

Lavandula coronopifolia Poir., 44, 54 App. pubescens Decne., 54 App.

Lectotypes, 185-187*

Lectotypification in Adelocaryum, 141-154* in Pedicularis, 57-64*, 185-187 in Rivea, 467-468*

Leguminosae, 54 App., 205 App.

Leontopodium (Pers.) R.Br., 235-264* sect. Alpina Hand.-Mazz., 256 sect. Leontopodium, 256 sect. Nobilia Hand.-Mazz., 257 alpinum Cass., 235-237, 246, 250-251, 253-254, 256 andersonii C.B.Clarke, 238, 250-253 artemisiifolium (H.Lév.) Beauverd, 238, 250-251, 254 aurantiacum, 255

caespitosum Diels, 238, 250-253, 257

calocephalum (Franch.) Beauverd, 238, 250-254, 256

dedekensii (Bureau \& Franch.) Beauverd, 238, 250-252, 254, 257

discolor Beauverd, 239, 250

franchetii Beauverd, 239, 250-251

haastioides Hand.-Mazz., 239, 250-251, 255

himalayanum DC., 239, 250-251, 254

jacotianum Beauverd, 239, 250-252, 254, 256

japonicum Miq., 239, 250-251

leontopodinum (DC.) Hand.-Mazz., 239, 250-251

leontopodioides (Willd.) Beauverd, 239, 250-254, 256

lingianum (Y.L.Chen) Dickoré, 235, 254 255

microphyllum Hayata, 240, 246, 250-254, 256-257

nanum Hand.-Mazz., 240, 250-251, $253-$ 254

nivale (Ten.) Huet ex Hand.-Mazz., 235236, 240, 246, 250-251, 253, 256 subsp. alpinum (Cass.) Greuter, 235 ochroleucum Beauverd, 240, 250-253, 256 pusillum (Beauverd) Hand.-Mazz., 240, 250-251, 253

sinense Hemsl., 241, 250-253, 257

souliei Beauverd, 241, 250-254, 256

stracheyi (Hook.f.) C.B.Clarke ex

Hemsl., 241, 250-253

Lepisanthes listeri King ex Radlk., 437

Leptadenia pyrotechnica (Forssk.) Decne., 203 App.

Leptoboea Benth., 177 multiflora (C.B.Clarke) Gamble, 175 subsp. grandifolia B.L.Burtt, 166

Lepus europaeus Pallas, 401

Leucas urticifolia (Vahl) R.Br. ex Sm., 205 App.

Leucogenes Beauverd, 248-249, 252-253, 255 grandiceps (Hook.f.) Beauverd, 244, 246, 250, 252, 254

leontopodium (Hook.f.) Beauverd, 245246, 250 
Leysera L., 247

gnaphalodes (L.) L., 245-246, 253-254

leyseroides (Desf.) Maire, 53 App.

Life history of Vaccinium, 7-24*

Limonium, 194, 393

axillare (Forssk.) Kuntze, 194-196 \&

Fig., 207 App.

cylindrifolium (Forssk.) Verdc. ex Cufod., 195, 207 App.

Lindelofia Lehm., 141-154* [143]

sect. Brandia M.Popov, 143

anchusoides (Lindl.) Lehm., 142-144

longiflora (Benth.) Baill., 141, 143

spectabilis Lehm., 141, 143

Lindenbergia

indica (L.) Kuntze, 56 App.

indica (L.) Vatke, 206 App.

Litostigma Y.G.Wei, F.Wen \& M.Möller, 161-184* [177 Fig., 178]

coriaceifolium Y.G.Wei, F.Wen \&

M.Möller, 161-162 \& Map, 167, 175,

177-181 [177 Fig., 179, 180 Fig.]

crystallinum Y.M.Shui \& W.H.Chen,

161-162 \& Map, 167, 175-177 \& Fig.,

179, 181 \& Fig.

LLEWELLYN, O.A., 25-35, 37-56, 189-208

Loranthaceae, 55 App.

Lotononis platycarpa (Viv.) Pic.Serm., 55 App.

Loxostigma

fimbrisepalum K.Y.Pan, 167, 175

griffithii (Wight) C.B.Clarke, 167, 175

Lycium

shawii Roem. \& Schult., 43, 46, 56

App.

Lycopodioides

dichotomum taxiforme, 72

Lysionotus D.Don, 178

chingii Chun ex W.T.Wang, 167, 175

forrestii W.W.Sm., 167, 175

pauciflorus Maxim., 167, 175

Lythraceae, 205 App.

Machilus

listeri King ex Hook.f., 437

Maerua

crassifolia Forssk., 52 App.

oblongifolia (Forssk.) A.Rich., 193, 204

App.
Malaysia, Peninsular, 75-121, 209-217*, 265-342* [329 Map]

Malvaceae, 55 App., 206 App.

Mandevilla Lindl., 1-2 subgen. Exothostemon (G.Don)

Woodson, 1

subgen. Mandevilla, 1

catimbauensis Souza-Silva, Rapini \& J.F.Morales, 1-5 [2, 3 Fig.] dardanoi M.F.Sales, Kin-Gouv. \&

A.O.Simões, 1-2, 4

leptophylla (A.DC.) K.Schum., 2, 4

matK, 235-264*

Matthiola

arabica Boiss., 42, 53 App.

longipetala (Vent.) DC., 53 App.

Mattiasrum

lambertianum (C.B.Clarke) Brand, 152

Maundia F.Muell., 358

Maytenus

parviflora (Vahl) Sebsebe, 193, 204 App. senegalensis (Lam.) Exell, 208 App.

somalensis (Loes.) Cufod., 208 App.

Mediterranean Floristic Region, 353-398*

Megaptera

novaeangliae Borowski, 190

Melanospora

brevirostris (Fuckel) Höhn., 417

Mellivora

capensis Schreber, 40

Menispermaceae, 206 App.

Mentha longifolia (L.) L., 54 App.

Mering, S. von, 353-398

Micrococca mercurialis (L.) Benth., 205 App.

Micromeria imbricata (Forssk.) C.Chr., 54 App.

Microparacaryum (Popov ex H.Riedl)

Hilger \& Podlech, 142

MilL, R.R., 141-154, 185-187

MilleR, A.G., 25-35, 37-56, 189-208

Molecular phylogeny of Leontopodium, 235-264*

MölLER, M., 161-184

Molluginaceae, 206 App.

Mollugo nudicaulis Lam., 206 App.

Moraceae, 55 App., 206 App.

Morales, J.F., 1-5 


\section{Morettia}

canescens Boiss., 53 App.

Morphology of Triglochin, 353-398*

Mucina, L., 353-398

Mucorales, 420

Myriophyllum spicatum L., 45, 54 App.

Myxochlamys A.Takano \& Nagam., 75, 347-352*

amphiloxa R.J.Searle, 347-352 [347, 348

Fig., 349 Map]

mullerensis A.Takano \& Nagam., 347, 351

Myxomycetes, 421

Nanorrhinum hastatum (R.Br. ex Benth.) Ghebr., 206 App.

Neotropics, $1-5^{*}$

Nepal, 57-64*, 451-465

Nepeta, 147 sheilae Hedge \& R.A.King, 43, 47, 54 App.

Nerium, 46-48 oleander L., 45-47 [45 Fig.], 52 App.

New combinations in Adelocaryum, 141-154* in Pedicularis, 185-187*

New genus of Gesneriaceae, 161-184

New Guinea, 431-450*

New series in Pedicularis, 185-187*

New species in Adelocaryum, 141-154*

in Begonia, 123-140*

in Boehmeria, 431-450

in Carpolobia, 343-346*

in Chassalia, 219-233

in Gentianaceae, 155-160

in Mandevilla, 1-5

in Myxochlamys, 347-352*

in Paraboea, 209-217

in Pedicularis, 185-187*

in Psychotria, 219-233

in Riedelia, 65-68*

in Roella, 425-430*

New status in Pedicularis, 57-64*

New taxa of Pedicularis, 185-187
New variety of Pedicularis, 185-187*

Newman, M.F., 65-68, 347-352, 451-465

Nothofagus, 9

Nothosaerva brachiata (L.) Wight, 194, 197, 203 App.

Novitates Gabonenses 74, 343-346

Nyctaginaceae, 206 App.

Ochradenus baccatus Delile, 42-44, 55 App., 207 App.

Oedera L., 247 squarrosa (L.) Anderb. \& K.Bremer, 245246, 253-254

Old World, 431-450

Oldenlandia corymbosa L., 208 App.

Oman, 25-35, 141-154*

Ophioglossaceae, 55 App.

Ophioglossum polyphyllum A.Braun, 55 App.

Opithandra primuloides (Miq.) B.L.Burtt, 167, 175

Orchidaceae, 55 App.

Oreocharis auricula (S.Moore) C.B.Clarke, 167, 175

Ornithoboea arachnoidea (Diels) Craib, 167, 175 wildeana Craib, 167, 175

Orobanchaceae, 55 App., 57-64*, 185-187, 206 App.

Orthosiphon pallidus Royle ex Benth., 205 App.

Oryctolagus, 401 cuniculus L., 401

Oryzopsis holciformis (M.Bieb.) Hack. subsp. abyssinica (Freitag) D.Heller, 44, 54 App.

Otostegia fruticosa (Forssk.) Schweinf. ex Penzig, 44, 46, 54 App.

Pakistan, 185-187*

Palawan, Philippines, 123-140*

Palmae, 55 App., 206 App.

Panaeolus antillarum (Fr.) Dennis, 408 409 campanulatus (L.) Quél., 409 fimiputris auct., 410 
papilionaceus (Bull.) Quél., 409

phalaenarum (Fr.) Quél., 410

retirugis (Fr.) Gillet, 409

semiovatis (Sowerby) S.Lundell \&

Nannf., 408-409

var. minor (J.E.Lange) Bon, 409

var. phalaenarum (Fr.) Ew.Gerhardt, 409

separatus (L.) Quél., 410

speciosus P.D.Orton, 410

sphinctrinus (Fr.) Quél., 409

subfirmus P.Karst., 410

Pancratium

maximum Forssk., 203 App.

Pandion

haliaetus L., 191

Panicum

coloratum L., 207 App.

turgidum Forssk., 54 App., 207 App.

Papua New Guinea, 431-450

Papua, Indonesia, 65-68*

Paraboea, 209-217*

acutifolia (Ridl.) B.L.Burtt, 168, 175

brunnescens, 213

caerulescens (Ridl.) B.L.Burtt, 216

capitata Ridl., 168, 175

crassifolia (Hemsl.) B.L.Burtt, 168, 175

gracillima Kiew, 209-213 [210 Map, 211

Fig.]

lambokensis Kiew, 209-210 \& Map, 213216 [214 Fig.]

laxa Ridl., 209, 212-213

rufescens (Franch.) B.L.Burtt

var. umbellata (Drake) K.Y.Pan, 168, 175

verticillata (Ridl.) B.L.Burtt, 213, 216

Paracaryopsis (H.Riedl) R.R.Mill, 141$154^{*}$

coelestina (Lindl.) R.R.Mill, 143-146

lambertiana (C.B.Clarke) R.R.Mill, 144, 152

malabarica (C.B.Clarke) R.R.Mill, 152

Paracaryum (A.DC.) Boiss., 141, 143-144

coelestinum (Lindl.) Benth. \& Hook.f., 146-147

intermedium (Fresen.) Hiliger \& Podlech, 41, 52 App.

lambertianum C.B.Clarke, 143, 152

malabaricum C.B.Clarke, 152

trinervium Duthie, 141
Paralagarosolen Y.G.Wei, 171-172, 176

fangianum Y.G.Wei, 168, 175

Parasola

misera (P.Karst.) Redhead, Vigalys \& Hopple, 407

Parietaria alsinifolia Delile, 56 App.

Paspalidium desertorum (A.Rich.) Stapf, 207 App.

Patzelt, A., 25-35

Pavonia arabica Hochst. ex Steud., 206 App.

Pedicularis L., 62, 185-187* sect. Phanerantha H.L.Li, 185

ser. Bicornutae R.R.Mill, 185

ser. Klotzschianae R.R.Mill, 185-186

ser. Macranthae Prain ex Hurus., 185-186

ser. Megalanthae (Prain) Bonati, 185

ser. Rhynchotrichae R.R.Mill, 185-186

ser. Tenuirostres Maxim., 185-186

unranked Macranthae Bunge, 186

unranked Macranthae Prain, 185-186

unranked Pectinatae Prain, 186

bicornuta Klotzsch, 185-186

var. adenocalyx R.R.Mill, 185-186

var. bicornuta, 186

bipinnatifida (Pennell) R.R.Mill, 185-186

elephantoides, 185

eximia G.Watt, 186

heteroglossa (Prain) Pusalkar \&

D.K.Singh, 58-62 [60 Fig.]

klotzschii Hurus., 186

macrantha Klotzsch, 186

murreeana R.R.Mill \& R.Bone, 185, 187

oederi Vahl, 59, 62

subsp. heteroglossa (Prain) Pennell, 5859

subsp. typica, 57

var. heteroglossa Prain, 57-64*

var. oederi, 58, 62

f. rubra (Maxim.) P.C.Tsoong, 62

pectinata Wall. ex Benth., 187

subsp. bipinnatifida Pennell, 186

subsp. palans (Prain) Pennell, 187

var. palans Prain, 187

pyramidata, 187

rhynchotricha P.C.Tsoong, 186

staintonii R.R.Mill, 185, 187

stewartii Pennell, 187

versicolor Royle, 61-62 
versicolor Wahlenb.

var. rubra Maxim., 62

Peganum

harmala L., 41, 56 App.

Pelicanus

rufescens Gmelin, 191

Pennisetum, 46

divisum (J.F.Gmel.) Henrard, 46, 54 App.

setaceum (Forssk.) Chiov., 42, 54 App.

setigerum (Vahl) Wipff, 207 App.

Pentatropis nivalis (J.F.Gmel.) D.V.Field \&

J.R.I.Wood, 193, 203 App.

Pernambuco State, Brazil, 1-5

Petrocodon Hance, 171, 173 dealbatus Hance, 168, 175

Petrocosmea Oliv., 161-184*

crystallina Y.M.Shui \& W.H.Chen, 161162, 175-176

kerrii Craib, 168, 175

minor Hemsl., 168, 175

nervosa Craib, 168, 175

sericea C.Y.Wu ex H.W.Li, 168, 175

\section{Peziza}

fimeti (Fuckel) Seaver, 414

stercorea Pers., 411

Pezizaceae, 414

Pezizales, 411-413

Phaeotrichiaceae, 419

Phenology

of Vaccinium, 7-24*

Philippines, 123-140

Phlomis

brachyodon (Boiss.) Zohary ex Rech.f., 43, 47, 54 App.

\section{Phoenix}

dactylifera L., 45 \& Fig., 55 App., 196, 206 App.

\section{Phomatospora}

coprophila M.J.Richardson, 418

minutissima (P.Crouan \& H.Crouan)

N.Lundq., 419

Phragmites, 47

australis (Cav.) Trin. ex Steud., 45 \& Fig., 54 App.

Phyllanthaceae, 206 App.

Phyllanthus

fraternus G.L.Webster, 206 App.

leucopyrus (Willd.) D.Koenig ex Roxb., 206 App. maderaspatensis L., 206 App.

rotundifolius Klein ex Willd., 206 App.

Phylogeny

of Gesneriaceae, 161-184*

Physaraceae, 421

Physarales, 421

Physarum didermoides (Ach. ex Pers.) Rostaf., 421

Pilaira

anomala Y.Ling, 420

moreaui Y.Ling, 420

Pilobolaceae, 420

Pilobolus

crystallinus (F.H.Wigg.) Tode

var. crystallinus, 420

var. kleinii (Tiegh.) R.Y.Zheng \&

G.Q.Chen, 421

roridus (Bolton) Pers.

var. umbonatus (Buller) F.M.Hu \&

R.Y.Zheng, 421

Piptatherum

holciforme (M.Bieb.) Roem. \& Schult., 54

App.

Piptocephalidaceae, 421

Piptocephalis, 421

repens Tiegh. \& G.Le Monn., 421

Pistacia

falcata Becc. ex Mart., 52 App.

khinjuk Stocks, 43, 52 App.

Plantaginaceae, 206 App.

Plastid markers, 235-264

Platystemma

violoides Wall., 168, 175

Pleosporales, 419

Pleuranthodium (K.Schum.) R.M.Sm., 65

Plicosepalus acaciae (Zucc.) Weins \& Polhill, 55 App.

Pluchea dioscoridis (L.) DC., 203 App.

Plumbaginaceae, 207 App.

Poa flabellata (Lam.) Raspail, 401

Poaceae, 207 App. see also Gramineae

Podosordaria ianthina J.C.Krug \& Cain, 418

Podospora, 416 australis (Speg.) Niessl, 416 communis (Speg.) Niessl, 416 curvula (de Bary ex G.Winter) Niessl, 416 
decipiens (G.Winter ex Fuckel) Niessl, 416

glutinans (Cain) Cain, 417

intestinacea N.Lundq., 416

perplexans (Cain) Cain, 416

setosa (G.Winter) Niessl, 416

Poecilolepis, 391

ficoidea (DC.) Grau, 393

Polycarpaea

repens (Forssk.) Asch. \& Schweinf., 204

App.

robbairea (Kuntze) Greuter \& Burdet, 52 App.

spicata Arn., 195, 204 App.

Polygala

erioptera DC., 207 App.

schwartziana Paiva, 43, 55 App.

Polygalaceae, 55 App., 207 App., 343-346*

Polygonaceae, 55 App.

Polypogon

viridis (Gouan) Breistr., 54 App.

Polysphaeria

subnudifax Verdc., 220

Porterandia Ridl., 265-342* [272 Fig., 285, 329 Map]

anisophylla auct. non (Jack ex Roxb.)

Ridl., 295-296, 313, 321

anisophylla (Jack ex Roxb.) Ridl., 265266, 268, 270-271, 273-278 [275, 277

Figs], 280-282, 284-285, 289-292 [290, 291 Fig.], 329 Map

var. macroptera (Miq.) Masam., 268, 312-313

var. polyneura (Vahl ex Winkl.)

Masam., 268, 305

beamanii Zahid, 270-271, 273-274, 278280, 284, 289, 293, 328, 330-331 \& Map

bruneiensis Zahid, 274, 278-280, 284, 289, 295, 329-332 \& Map

catappifolia auct. non Ridl., 303

catappifolia Ridl., 274, 278-280, 284, 288, 296, 301, 314, 329-331 \& Map

celebica Zahid, 265, 274, 278, 280, 284, 286, 289, 297, 328-329 \& Map

chanii Zahid, 270-271, 274, 278-280, 284, 289, 298, 329-331 \& Map

congestiflora Zahid, 274-277 [275 Fig.], 279-280, 284, 287, 300, 329-330 \& Map crosbyi (Burkill) A.C.Sm. \& S.Darwin, 267, 269, 285

dinghoui Zahid \& K.M.Wong, 265, 274, 276-279, 283, 287, 301-302 \& Fig., 329-331 \& Map, 341 App.

glabrifolia Ridl., 274, 277-280, 284, 288, 296, 303-304 \& Fig., 328, 330-331 \& Map

grandifolia Ridl., 274, 277-278, 280-281, 284, 288, 305-306 \& Fig., 308, 312, 325, 330-331 \& Map

hosei (Merr.) Zahid, 274, 278-279, 281, 284, 289, 308, 314, 328, 330-331 \& Map

kalimantanensis Zahid, 274, 278-279, 281, 284, 288, 309, 330-331 \& Map

kelantansis Tirveng., 328

lambirensis Zahid, 274, 278-281, 284, 288, 310, 329-332 \& Map

laxiflora Zahid, 274, 277-279, 281, 284, 287, 311, 329-332 \& Map

macroptera (Miq.) Tirveng., 274, 279, 281, 284, 289-290, 312, 330-331 \& Map

minor Ridl., 274, 281, 284, 289, 296, 308, 314, 325, 329-331 \& Map mussaendoides (Craib) Tirveng., 327 pauciflora Ridl., 274, 276-282, 284-285, 287, 315-316 \& Fig., 329-332 \& Map postarii Zahid, 270-271, 274-282 [275, 279 Figs], 284-285, 287, 317, 328, 330331 \& Map

puffii Zahid, 274, 277, 279-281, 284, 287, 319, 328, 330-331 \& Map

rarissima Zahid, 274, 277, 279, 283, 288, 320, 330-332 \& Map, 341 App.

scortechinii (King \& Gamble) Ridl., 265, 268, 270-274 [272 Fig.], 276 \& Fig., 278-282, 284, 287, 290, 321-323 \& Figs, 329 Map, 332

sericantha (W.C.Chen) W.C.Chen, 327 sessiliflora Ridl., 268, 285, 327 subsessilis auct. non (Valeton) Ridl., 295 subsessilis (Valeton ex Winkl.) Ridl., 268, 274, 276-278, 280-281, 284, 288, 325326 \& Fig., 329-330 \& Map tenuiflora (A.C.Sm.) A.C.Sm. \& S.Darwin, 267, 285

Portulaca oleracea L., 207 App. 
Portulacaceae, 207 App.

Posoqueria anisophylla Jack ex Burkill, 290

Pouzolzia sanguinea (Blume) Merr., 440 var. fulgens (Wedd.) Hara, 440

\section{Premna} resinosa (Hochst.) Schauer, 205 App.

Primula listeri King ex Hook.f., 437

Primulina tabacum Hance, 168, 175

Prismatocarpus alpinus (Bond) Adamson, 429

Priva adhaerens (Forssk.) Chiov., 208 App.

Prosopis, 198-200 cineraria (L.) Druce, 199 juliflora (Sw.) DC., 189, 193, 198-200 [199 Fig.], 205 App.

Psathyrella falklandica Cotton, 399

Pseudochirita W.T.Wang, 178

Pseudognaphalium Kirp., 248, 255 luteoalbum (L.) Hilliard \& B.L.Burtt, 245-246, 249-250

\section{Psilocybe} coprophila (Bull.) P.Kumm., 410 cubensis (Earle) Singer, 410 merdaria (Fr.) Ricken, 410 moelleri Guzmán, 410 subcoprophila (Britzelm.) Sacc., 410

Psychotria L., 219-233*, 331 subgen. Psychotria, 219, 228 subgen. Tetramerae (Hiern) E.M.A.Petit, 219, 229, 232

sect. Involucratae E.M.A.Petit \& Verdc., 219, 228

auxopoda E.M.A.Petit, 220

gossweileri E.M.A.Petit, 232

leptophylla Hiern, 219, 229, 232

mortehanii De Wild., 228

mwinilungae Verdc., 226, 228

nodiflora O.Lachenaud \& D.J.Harris, 219, 224-229 [225 Fig., 226 Map]

pteropus O.Lachenaud \& D.J.Harris, 219, 227, 229-233 [230 Fig., 231 Map] schnellii (Aké Assi) Verdc., 226, 228 Pteridophyta, 69-73

Pterocephalus brevis Coult., 43, 47, 53 App.

Pterogaillonia calycoptera (Decne.) Lincz., 43, 56 App.

Pulicaria crispa (Forssk.) Oliv., 41, 53 App. jaubertii E.Gamal-Eldin, 203 App.

Pusalkar, P.K., 57-64

Pycnocycla tomentosa Decne., 56 App.

Pyronemataceae, 411

Qaraqir, Saudi Arabia, 37-56* [39 Map]

Quercus

listeri King, 437

Ramonda Rich., 174, 176-177

myconi (L.) Rchb., 162, 168, 175

Randia auct. non Houst. ex L., 285

Randia Houst. ex L., 266-267, 269 sect. Anisophyllea Hook.f., 268, 285

aculeata L., 266

anisophylla (Jack ex Roxb.) Hook.f., 266, 268, 285, 290

beccarii King \& Gamble, 307

mitis L., 266

mussaendoides Craib, 328

scortechinii auct. non King \& Gamble, 296, 314

scortechinii King \& Gamble, 268, 285, 321

sericantha W.C.Chen, 268

Ranunculaceae, 55 App.

Raphiocarpus Chun, 178 begoniifolius (H.Lév.) B.L.Burtt, 169, 175-176 petelotii (Pellegr.) B.L.Burtt, 169, 175176

RAPINI, A., 1-5

Rare species in Saudi Arabia, 189-208* of Selaginella, 69-73

Red Sea, 189

Refuge, 37-56*

Relhania L'Hér., 247 calycina L'Hér., 245-246, 253-254

Relict species, 37-56*

Reseda aucheri Boiss. var. bracteata (Boiss.) Abdallah \& De Wit, 55 App. 
pentagyna Abdallah \& A.G.Mill., 46-47, 55 App.

Resedaceae, 55 App., 207 App.

Retama raetam (Forssk.) Webb \& Berth., 42, 44, 46, 55 App.

Revisions

of Begonia, 123-140

of Cautleya, 451-465*

of Triglochin, 353-398*

Rhabdothamnopsis Hemsl., 178 sinensis Hemsl., 169, 175

Rhamnaceae, 55 App., 207 App.

Rhizophora, 192, 197 mucronata Lam., 192-193 \& Fig., 200, 207 App.

Rhizophoraceae, 207 App.

Rhynchosia minima (L.) DC., 205 App.

Rhynchotechum discolor (Maxim.) B.L.Burtt, 169, 175 parviflorum Blume, 169, 175

Richardson, M.J., 399-423

Riedelia Oliv., 65-68* subgen. Schefferia Valeton, 65, 68 sect. Cornuta Valeton, 65 sect. Geocharides Valeton, 68 sect. Macrantha Valeton, 65, 68 charontis M.F.Newman, 65, 66-68 [66 Fig.] tenuifolia Valeton, 65, 68

Rivea Choisy, 467-468 bernoulliana (Peter) Hallier f., 467-468

Roella L., 425-430* ser. Ciliatae Adamson, 425 ser. Muscosae Adamson, 425 ser. Prostratae Adamson, 425, 429 ser. Roella, 425 ser. Spicatae Adamson, 425 ser. Squarrosae Adamson, 425 goodiana, 428-429 insizwae (Zahlbr.) Hilliard \& B.L.Burtt, 425 muscosa L.f., 425, 428-429 recurvata, 428-429 uncinata Cupido, 425-429 [426-427 Figs, 428 Map]

Roscoea, 452 sect. Cautlea Royle ex Benth., 451, 454 elatior Sm., 455 gracilis $\mathrm{Sm} .$, 451-452, 455 lutea Royle, 455

lutea Sm., 451-452

petiolata, 464

spicata Sm., 451, 460

Rubiaceae, 55 App., 208 App., 219-233*, 265-342*

subfam. Cinchonoideae, 269

subfam. Ixoroideae, 269

tribe Aulacocalyceae, 269

tribe Coffeeae, 269

tribe Gardenieae A.Rich. ex DC., 265$342 *$

tribe Hypobatheae, 269

tribe Pavetteae, 269

tribe Psychotrieae, 219

tribe Vanguerieae, 269

Rubite, R.R., 123-140

Rumex

nervosus Vahl, 44, 55 App.

pictus Forssk., 55 App.

vesicarius L., 42, 55 App.

Rutaceae, 56 App.

Ryparobius spegazzinii Gamundi, 415

Saccharum ravennae (L.) Murr., 45 \& Fig., 54 App.

\section{Saccobolus}

beckii Heimerl, 413

depauperatus (Berk. \& Broome) Rehm, 413

quadrisporus Massee \& E.S.Salmon, 412413

verrucisporus Brumm., 413

versicolor (P.Karst.) P.Karst., 413

Sageretia thea (Osbeck) M.C.Johnst., 42, 55 App.

Saintpaulia Wendl., 177 tongwensis B.L.Burtt, 169, 175 velutina B.L.Burtt, 169, 175

Salsola tetrandra Forssk., 42, 52 App.

Salvadora persica L., 193-194, 196, 208 App.

Salvadoraceae, 208 App.

Salvia, 452 palaestina Benth., 43, 47, 54 App.

SAmuel, R., 235-264

Sarcocornia fruticosa (L.) A.J.Scott, 363 
tegetaria S.Steffen, Mucina \&

G.Kadereit, 391

Satureja

thymbrifolia Hedge \& Feinbrun, 43, 47, 54 App.

Saudi Arabia, 25-35, 37-56* [39 Map]

Farasan Archipelago in, 189-208* [190 Fig.]

Scandix

stellata Banks \& Sol., 42, 56 App.

Scaphochlamys Baker, 75-121* [79 Fig., 80], 347

abdullahii Y.Y.Sam \& Saw, 82, 84-85 Map

anomala (Hallier f.) R.J.Searle, 75, 82,

85-86 Fig., 94 Map

argentea R.M.Sm., 82, 87, 94 Map atroviridis Holttum, 81, 88-89 Map biloba (Ridl.) Holttum, 81, 85 Map, 88, 90 var. lanceolata (Ridl.) Holttum, 75, 89 breviscapa Holttum, 82, 91-92 Map, 117 burkillii Holttum, 84-85 Map, 92 calcicola A.D.Poulsen \& R.J.Searle, 81, 93-94 Map

concinna (Baker) Holttum, 82, 92 Map, 94, 118

cordata Y.Y.Sam \& Saw, 81, 85 Map, 95 erecta Holttum, 79 Fig., 83-84, 89 Map, 96

gracilipes (K.Schum.) S.Sakai \& Nagam., 81, 94 Map, 97

grandis Holttum, 84-85 Map, 97

klossii (Ridl.) Holttum, 92 Map, 98

var. glomerata Holttum, 83-84, 99

var. klossii, 84, 98

var. minor Holttum, 84, 100

kunstleri (Baker) Holttum, 100, 102 Map

var. kunstleri, 83, 101, 103

var. rubra (Ridl.) Holttum, 83, 102

var. speciosa C.K.Lim, 83, 103

lanceolata (Ridl.) Holttum, 84, 102 Map, 103

laxa Y.Y.Sam \& Saw, 81, 85 Map, 104 105

longifolia Holttum, 75, 90

malaccana Baker, 79-80, 83, 92 Map, 105-106

mat-kilau C.K.Lim, 75, 104

minutiflora Jenjitt. \& K.Larsen, 83, 89 Map, 106 obcordata Sirirugsa \& K.Larsen, 83-84,

89 Map, 107

oculata (Ridl.) Holttum, 82, 102 Map,

108

pennipicta Holttum, 82, 102 Map, 109

perakensis Holttum, 83, 92 Map, 109

petiolata (K.Schum.) R.M.Sm., 82, 110

111 Map, 118

polyphylla (K.Schum.) B.L.Burtt \&

R.M.Sm., 83, 111 \& Map

reticosa (Ridl.) R.M.Sm., 80, 82, 111

Map, 112

rubescens Jenjitt. \& K.Larsen, 82, 89

Map, 113

rubromaculata Holttum, 83, 102 Map,

114

subbiloba (Burkill ex Ridl.) Holttum, 83, 92 Map, 115

sylvestris (Ridl.) Holttum, 82, 89 Map,

116

tenuis Holttum, 83, 102 Map, 116

Schimpera

arabica, 42

Schizothecium

conicum (Fuckel) N.Lundq., 416

dakotense (Griffiths) N.Lundq., 417

glutinans (Cain) N.Lundq., 417

tetrasporum (G.Winter) N.Lundq., 417

vesticola (Berk. \& Broome) N.Lundq., 417

Schumanniophyton Harms, 269

Schweinfurthia pedicellata Benth. \& Hook.f., 206 App. pterosperma (A.Rich.) A.Braun, 208

App.

ScotLand, R.W., 69-73

Scrophulariaceae, 56 App., 63, 208 App.

SEARLE, R.J., 75-121, 347-352

Seddera

latifolia Hochst. \& Steud., 204 App.

virgata Hochst. \& Steud., 204 App.

Selaginella P.Beauv.

arroyoana M.Kessler \& A.R.Sm., 69-73*

[71 Fig., 72 Map]

chiquitana M.Kessler, A.R.Sm. \&

M.Lehnert, 69, 71-72

falcata (P.Beauv.) Spring, 71-72

kochii Hieron., 71

xiphophylla Baker, 71

Selaginellaceae, 69-73 


\section{Selinia}

pulchra (G.Winter) Sacc., 418

Semi-arid regions, Brazil, 1-5

Senecio

glaucus L., 42, 53 App.

Senna alexandrina Mill., 205 App.

holosericea (Fresen.) Greuter, 205 App. italica Mill., 46, 55 App., 205 App.

Senra incana Cav., 206 App.

Setaria verticillata (L.) P.Beauv., 207 App. viridis (L.) P.Beauv., 207 App.

Shorea curtisii Dyer ex King, 109

Shui, Y.-M., 161-184

Siamanthus K.Larsen \& Mood, 65

Silene grisea Boiss., 52 App.

SingH, D.K., 57-64

Sino-Himalayan flora, 235-264*

Sinoleontopodium Y.L.Chen, 235-264* lingianum Y.L.Chen, 241, 249-251, 254

Sisymbrium erysimoides Desf., 42, 53 App.

Solanaceae, 56 App., 208 App.

Solanum coagulans Forssk., 208 App. forskalii Dunal, 208 App. surattense Burm.f., 208 App. thruppii C.H.Wright, 208 App. virginianum L., 208 App.

Solidago L., 248 rugosa Mill., 245-246, 253

\section{Sordaria}

fimicola (Roberge ex Desm.) Ces. \& de Not., 417

macrospora Auersw., 417

superba de Not., 417

Sordariales, 415-416

South Africa, 353-398*, 425-430*

Southeast Asia, 75-121*

Southern hemisphere, 399-423*

SouzA-SiLva, R.F. DE, 1-5

Spelaeanthus

chinii Kiew, A.Weber \& B.L.Burtt, 169, 175

Sphaerodes

fimicola (E.C.Hansen) P.Cannon \&

D.Hawksw., 417
Spores, 69-73*

Sporobolus

helvolus (Trin.) T.Durand \& Schinz, 207

App.

ioclados (Trin.) Nees, 208 App.

spicatus (Vahl) Kunth, 207 App.

Sporormiaceae, 419

Sporormiella, 419

australis (Speg.) S.I.Ahmed \& Cain, 419

grandispora S.I.Ahmed \& Cain ex

J.C.Krug, 419

intermedia (Auersw.) S.I.Ahmed \& Cain ex Kobayasi, 419

lageniformis (Fuckel) S.I.Ahmed \& Cain ex Kobayasi, 420

leporina (Niessl) S.I.Ahmed \& Cain, 420

megalospora (Auersw.) S.I.Ahmed \& Cain, $\mathbf{4 2 0}$

minima (Auersw.) S.I.Ahmed \& Cain, $\mathbf{4 2 0}$

ovina (Desm.) S.I.Ahmed \& Cain, $\mathbf{4 2 0}$

systenospora S.I.Ahmed \& Cain, $\mathbf{4 2 0}$

Stachys

aegyptiaca Pers., 43-44, 47, 54 App.

StAPLES, G.W., 467-468

Stenella

attenuate Gray, 190

longirostris Gray, 190

Stipa

arabica, 54 App.

Stipagrostis

ciliata (Desf.) De Winter, 54 App.

foëxiana (Maire \& Wilczek) De Winter, 43, 54 App.

obtusa (Delile) Nees, 54 App.

plumosa (L.) Munro ex T.Anderson, 43, 54 App.

Streptocarpus Lindl., 176-177 andohahelensis Humbert, 169, 175

beampingaratrensis Humbert, 169, 175

dunnii Hook.f., 169, 175

hilsenbergii R.Br., 169, 175

holstii Engl., 170, 175

ibityensis Humbert, 170, 175

papangae Humbert, 170, 175

rexii Lindl., 170, 175

saxorum Engl., 170, 175

Striga

gesnerioides (Willd.) Vatke, 206 App.

Strix

butleri Hume, 41 


\section{Stropharia}

semiglobata (Batsch) Quél., 411

Strophariaceae, 410

Stuessy, T.F., 235-264

Suaeda, 190 aegyptiaca (Hasselq.) Zohary, 203 App. fruticosa Forssk. ex J.F.Gmel., 194, 203 App.

monoica Forssk. ex J.F.Gmel., 194, 203 App.

vermiculata Forssk. ex J.F.Gmel., 203 App.

Sukunia A.C.Sm., 267

Sulawesi, 265-342* [329 Map]

Sumatra, 75-121, 265-342* [329 Map], 431-450*

Sundaland, 265-342*

Sylvilagus

floridanus J.A.Allen, 401

Symphyotrichum Nees, 248

novae-angliae (L.) G.L.Nesom, 245-246, 254

Synopses

of Adelocaryum, 141-154

of Rivea, 467-468

Syringodium

isoetifolium (Asch.) Dandy, 195, 204 App.

Tamaricaceae, 56 App., 208 App.

Tamarix L., 48, 208 App.

aphylla (L.) H.Karst., 46, 56 App. nilotica (Ehrenb.) Bunge, 45, 56 App.

Tanacetum

sinaicum (Fresen.) Delile ex K.Bremer \& Humphries, 53 App.

Taverniera cuneifolia (Roth) Arn., 205 App. lappacea (Forssk.) DC., 205 App.

Taxonomy of Boehmeria, 431-450*

of Cautleya, 451-465*

of Chassalia, 219-233*

of Mandevilla, 1-5*

of Porterandia, 265-342*

of Psychotria, 219-233*

of Selaginella, 69-73*

of Triglochin, 353-398*

Taxus

baccata L., 72

Tephrosia purpurea (L.) Pers., 205 App.

subtriflora Hochst. ex Baker, 205 App.

uniflora Pers., 205 App.

Tetraena, 190

alba (L.f.) Beier \& Thulin, 208 App.

boulosii (A.I.Hosny) M.Hall, 200, 208 App.

coccinea (L.) Beier \& Thulin, 208 App.

simplex (L.) Beier \& Thulin, 193-195, 208

App.

Tetraphyllum, 177

Tetrapogon

tenellus (K.D.Koenig ex Roxb.) Chiov., 207 App.

Teucrium leucocladum Boiss., 44, 46-47, 54 App. popovii R.A.King, 54 App.

Thailand, 75-121, 155-160, 209-217*, 431$450 *$, 451-465

Thalassia hemprichii Ehrenb. ex Solms, 195, 205 App.

Thalassodendron ciliatum (Forssk.) Hartog, 195, 204 App.

Thelebolaceae, 414

Thelebolales, 414

Thelebolus, 414-415

crustaceus (Fuckel) Kimbr., 415

microsporus, 414

nanus Heimerl, 414

polysporus (P.Karst.) Otani \& Kanzawa, 414

stercoreus Tode, $\mathbf{4 1 4} 415$

Thomas, Z., 431-450

Tibet, 235-264

Timonius hosei Merr., 308

Trianthema portulacastrum L., 202 App.

sheilae A.G.Mill. \& J.A.Nyberg, 202 App.

Trichodelitschia

bisporula (P.Crouan \& H.Crouan) Munk, 419

munkii N.Lundq., 419

Tricholaena

teneriffae (L.f.) Link, 54 App., 207 App.

Tricholomataceae, 404

Triglochin L., 353-398* [358]

atlantica Willd. ex Kunth, 363

barrelieri Loisel., 353-355, 360 Fig., 361

Map, 362-364, 369, 394-395

var. maura Pau, 355, 363 
buchenaui Köcke, Mering \& Kadereit, 353, 357 Fig., 360 Fig., 362, 384 Map, 389, 391-394 [392 Fig.]

bulbosa L., 353-398 [370]

subsp. barrelieri (Loisel.) Rouy, 355, 357, 362

subsp. bulbosa, 353-354, 357, 359, 364,

371 Fig., 372 Map, 373-374, 385, 394

subsp. calcicola Mering, Köcke \&

Kadereit, 353-354, 357 Fig., 359,

371-374 [371 Fig., 372 Map],

376-378 [377 Fig.], 394-395

subsp. laxiflora (Guss.) Rouy, 355, 357, 368

subsp. maura (Pau) Horn, 357

subsp. quarcicola Mering, Köcke \&

Kadereit, 353-354, 357 Fig., 371-

374 [371 Fig., 372 Map], 379-381

[380 Fig.], 394-395

subsp. tenuifolia (Adamson) Horn,

353-354, 357, 371-374 [371 Fig., 372

Map], 381-382, 394

var. genuina Maire, 363

var. maura (Pau) Maire, 355, 363

$\beta$ robustior Roem. \& Schult., 371

compacta Adamson, 353-355, 357 \& Fig., 359-360 \& Fig., 362, 369, 373, 382-386 [383, 384 Map], 394-395

elongata Buchenau, 353-355, 357 \& Fig., 360 Fig., 362, 369, 384 Map, 388-389, 393-394

laxiflora Guss., 353-355, 360 Fig., 361

Map, 362, 364, 368-369, 394-395

maritima L., 354, 358-359

milnei Horn, 353-354, 357, 359-360 \&

Fig., 362, 385-388 [386, 388 Map], 394

palustre Desf., non L., 368

palustris L., 354-355, 358, 363-364

patens Steud., 371

striata Ruiz \& Pav., 354, 357, 359

tenuifolia Adamson, 355, 357, 373, 381382

Trigonella

stellata Forssk., 42, 55 App.

Tristemon Raf., 358

$\operatorname{trn} \mathrm{L} / \mathrm{F}, 235-264 *$

Tropical zone, 7-24*

Trukia Kaneh., 269

Tursiops

truncatus Montagu, 190
Typha, 47

domingensis Pers., 45 \& Fig., 56 App.

Typhaceae, 56 App.

Typification

of Triglochin, 353-398*

Ulex

europaeus L., 403

Umbelliferae, 56 App.

Umbilicus horizontalis (Guss.) DC., 53 App.

Uragoga mortehanii De Wild., 224

Urticaceae, 56 App., 208 App., 431-450*

Vaccinium, 7-24*

sect. Baccula-nigra, 11

sect. Batodendrin, 11

sect. Calcicolus, 10

sect. Ciliata Nakai, 11, 13

sect. Conchophyllum, 10

sect. Cyanococcus A.Gray, 10-11, 13, 19

sect. Euepigynium Schltr., 8, 10

sect. Galeopetalum, 10

sect. Hemi-myrtillus, 10-11

sect. Herpothamnus, 11

sect. Myrtillus, 10-11, 19

sect. Nesococcus Copel., 8, 10

sect. Oreades, 10

sect. Oxycoccoides, 11

sect. Oxycoccus, 11

sect. Polycodium, 11

sect. Pyxothamnus, 10-11

sect. Vaccinium, 10

sect. Vitis-idaea, 11

acrobracteatum K.Schum., 9-10

angustifolium Aiton, 11

arboreum Marshall, 11

arctostaphylos L., 11

auriculifolium Sleumer, 10

boreale I.V.Hall \& Aalders, 10

calycinum $\mathrm{Sm} ., 10$

cereum G.Forst., 9-10

cespitosum Michx., 10

consanguineum Klotzsch, 10, 18

corymbosum L., 11, 18-19

crassifolium Andrews, 11

cylindraceum $\mathrm{Sm} ., 10$

darrowii Camp., 9, 11

deliciosum Piper, 10 
dentatum Sm., 10

erythrocarpum Michx., 11

fragile Franch., 11

gaultheriifolium (Griff.) Hook.f. ex

C.B.Clarke, 7, 10

hirsutum Buckley, 11

hirtum Thunb., 11, 18

horizontale Sleumer, 7, 10

macrocarpon Aiton, 7, 11

membranaceum Douglas ex Torr., 10

meridionale Sw., 8, 10, 15

myrsinites Lam., 11

myrtilloides Michx., 11

myrtillus L., 10, 20

myrtoides (Blume) Miq., 7, 10

oldhamii Miq., 11

ovalifolium Sm., 9-10

ovatum Pursh, 7, 11

oxycoccus L., 11

padifolium Sm., 11, 18

pallidum Aiton, 11, 18

parvifolium Sm., 11, 19

poasanum Donn.Sm., 10

praestans Lamb., 10

pseudotonkinense Sleumer, 10, 18

reticulatum $\mathrm{Sm} .$, 7, 10, 15

ridleyi Sleumer, 10

scoparium Leiberg ex Coville, 9-10

smallii A.Gray, 11

stamineum L., 11

tenellum Aiton, 11

uliginosum L., 7-8, 10, 20

varingiifolium (Blume) Miq., 8, 10

vitis-idaea $\mathrm{L} ., 11$

Vahlia

digyna (Retz.) Kuntze, 208 App.

Vahliaceae, 208 App.

Vale do Catimba National Park, Brazil, 1-2

Valerianaceae, 56 App.

Valerianella

oxyrhyncha Fisch. \& C.A.Mey., 43, 47, 56 App.

VANDER KLOET, S.P., 7-24

Verbascum, 56 App. deserticola (Murb.) Hub.-Mor., 46-47, 56 App.

sheilae Kit Tan, 45-46, 56 App.

sinaiticum Benth., 45-47, 56 App.

Verbenaceae, 208 App.

Vietnam, 155-160*, 451-465
Vitaceae, 208 App.

Volutella, 420

ciliata (Alb. \& Schwein.) Fr., 420

Vulpes

vulpes L., 40

WATLING, R., 399-423

WeI, Y.-G., 161-184

WeN, F., 161-184

Wentsaiboea D.Fang \& D.H.Qin, 171, 173, 176

renifolia D.Fang \& D.H.Qin, 170, 175

Western Cape, 425-430*

Wilmot-Dear, C.M., 431-450

WoNG, K.M., 265-342

Wood, J.R.I., 69-73

Xanthorrhoeaceae, 208 App.

Xylariaceae, 418

Xylariales, 418

Yemen, 25-35

Yunnan, 431-450

ZAHID, M.S., 265-342

Zaleya

pentandra (L.) C.Jeffrey, 202 App.

Zehneria

anomala C.Jeffrey, 204 App.

Zilla

spinosa (L.) Prantl, 42, 44, 53 App.

Zingiberaceae, 65-68*, 75-121*, 347-352*, 451-465*

subfam. Alpinioideae Link, 65

subfam. Zingiberoideae, 451

tribe Riedelieae W.J.Kress, 65

tribe Zingibereae Misn., 75-121*, 347

Ziziphus

spina-christi (L.) Desf., 193, 196, 207 App.

Zoega

purpurea Fresen., 53 App.

Zoopagales, 421

Zygomycota, 420

Zygophyllaceae, 56 App., 208 App.

Zygophyllum

album L.f., 208 App.

boulosii A.I.Hosny, 208 App.

coccineum L., 208 App.

simplex L., 208 App. 